PART II

FLATTENED SYSTEMS 


\title{
CROSSFERTILIZATION BETWEEN PLASMA, STELLAR DYNAMICS AND HYDRODYNAMICS
}

\author{
M. R. FEIX
}

Groupe de Recherches Ionosphériques, Université d'Orléans, Orléans, France

We present results on four different mediums characterised by their 'density conservation' in a two dimensional space (phase space for unidimensional plasma and self gravitating systems, configuration space for two dimensional Navier Stokes fluid and guiding center rod plasma).

We review the different problems (purely collective versus individual) and introduce the distinction between Lagrangian schemes (superparticles) and Eulerian schemes.

The steady states structures for these fluids and their open (plasma, hydrodynamic) versus closed (stellar system, accelerator beam, hydrodynamic) topologies are discussed. We then turn to their stability properties and show the condition for the presence of marginal adiabatic mode. The 'double water bag' system is fully studied and interesting analogies for completely trapped systems (accelerator beam and self gravitating systems) are pointed out.

\section{Introduction}

From the experimenter's point of view mediums like galaxies, plasmas and regular fluids look completely different. For the theoretician the problems look much more similar and the computational physicist can't resist to see what happens if he changes the sign of the density in Poisson law! The purpose of this paper is to compare the properties of different mediums, the mathematical structures of the problems and the different methods used to solve them. It is hoped that astronomers and plasma physicist will look onto one each other problems and solutions and that some crossfertilization will result.

\section{The Different Mediums}

There is no point here in describing the stellar dynamics systems! Plasma Physics systems are now well known by the astronomers. Let us point out that accelerator beams provide model of one species plasma with all particles trapped and boundaries limits quite similar to the stellar self gravitating systems. Of course there must be a focusing device to balance the space charge repulsion. Plasma and self gravitating systems form what we will call phase space fluids to remind that their evolution should be described in phase space.

Now we consider two new mediums. The first is a regular, incompressible and inviscid fluid described by the Navier Stokes equation. Moreover we restrict our studies to bidimensional fluids. We will see why in a moment. Navier Stokes equations can be 
written in the $x, y$ space

$$
\begin{aligned}
& \frac{\partial \mathbf{u}}{\partial t}+(\mathbf{u} \cdot \boldsymbol{\nabla}) \mathbf{u}+\frac{1}{\varrho} \nabla p=0 \\
& \boldsymbol{\nabla} \cdot \mathbf{u}=0,
\end{aligned}
$$

where $\varrho$ is the constant density. We introduce the vorticity $\boldsymbol{\omega}=\boldsymbol{\nabla} \times \mathbf{u}$. Due to the $x, y$ space dependence of the problem $\omega$ has only the third (along $z$ ) component different from zero. We call $\xi$ that component. Taking the curl of (1) it is easily shown that $\xi$ obeys

$$
\frac{\partial \xi}{\partial t}+\nabla \cdot(\xi \mathbf{u})=\frac{\partial \xi}{\partial t}+\mathbf{u} \cdot \nabla \xi=0
$$

Since $\boldsymbol{\nabla} \cdot \mathbf{u}=0$ we can deduce the two components of the velocity from a scalar potential $\Phi$ with

$$
\mathbf{u}=\frac{\partial \Phi}{\partial y} \mathbf{e}_{x}-\frac{\partial \Phi}{\partial x} \mathbf{e}_{y}
$$

where $\mathbf{e}_{x}, \mathbf{e}_{y}, \mathbf{e}_{z}$ are the unit vectors along $o x, o y, o z$. Taking into account $\xi e_{z}=\boldsymbol{\nabla} \times \mathbf{u}$ and Equation (3) we get

$$
\Delta \Phi+\xi=0 \text {. }
$$

Equations (2) and (4) are now the new 'model equation' for hydrodynamics. Equation (2) indicates that $\xi$ is a constant along the trajectory. This trajectory can be deduced from a fictious 'Electric field' $\mathbf{E}$ with $E_{x}=-\partial \phi / \partial x$ and $E_{y}=-\partial \phi / \partial y$ where $\Phi$ satisfies the usual Poisson equation. The only difference is that the dynamics of the vortex elements is given by $U_{x}=-E_{y}$ and $U_{y}=E_{x}$ instead of the usual $\dot{\mathbf{U}}=(\mathrm{e} / \mathrm{m}) \mathbf{E}$. These properties have been extensively used by Zabusky and Deem (1971) and by Christiansen and Zabusky (1973) to compute the nonlinear evolution of bidimensional fluids and formation of 'Vortex Streets'. In these calculations the Lagrangian point of view was used consisting in representing the vortex density $\xi$ by many points carrying each an elementary 'charge of vorticity' (eventually with a negative sign). One of the advantage of such a method is the possibility of using fast 'Poisson solver' algorithm developped for plasma and stellar dynamics studies.

Another interesting bidimensional medium is a plasma immersed in a very strong magnetic field, homogeneous along the direction of this field. The transverse motion is studied. In that case the velocity of a particle is given by the so called 'drift approximation' with

$$
\mathbf{u}=\frac{\mathbf{E} \times \mathbf{B}}{B^{2}}
$$

which can be written $U_{x}=E_{y} / B, U_{y}=-E_{x} / B$. Except for the trivial factor $(-1 / B)$ these are exactly the motion equation of the vortex elements considered above. On the 
other hand the continuity equation can be written

$$
\frac{\partial n}{\partial t}+\nabla \cdot n \mathbf{u}=0
$$

From the fact that $E=-\nabla \Phi$ and taking into account (5) we deduce that $\nabla \cdot \mathbf{u}=0$ and consequently

$$
\frac{\partial n}{\partial t}+\mathbf{u} \cdot \nabla n=0
$$

which is the equivalent of (2) with a conservation of the density along the trajectories in the $x, y$ space. Of course $\Phi$ satisfies the Poisson equation

$$
\Delta \Phi+e n=0
$$

in strict identity with the vorticity Poisson equation (4).

The fundamental property of these 4 mediums (one dimensional plasma, one dimensional stellar system, bidimensional fluid and plasma) is that in the two dimensional space describing the evolution we have conservation of the density $\{\xi(x, y) n(x, y)$ or $f(x, v)\}$. The other fundamental property is that the dynamics is obtained through a potential $\Phi$ obeying Poisson law. Both Eulerian or Lagrangian schemes can be used. By Langrangian scheme we mean representing the density by many 'elementary particles' each carrying a constant elementary 'charge' and 'mass'. We can also use Eulerian scheme. Although we will come back to the duality of these two schemes later on we point out a general discussion of the different models in Feix (1971).

\section{Problems}

\subsection{Collisionless verSUS COllisional REGIME}

The first level of description for plasmas and stellar dynamics systems is obviously the Boltzmann 'collisionless' equation (called Vlasov by the plasma physicists). This equation describes the 'violent relaxation' of stellar systems. In plasma also it describes the fast evolution (especially the different instabilities) and more studies are needed in its nonlinear solution. The final state could be the one predicted on statistical argument by Lynden Bell although computer studies have shown that the mixing of the phase space elements is not always complete and that bunch of particles do not obey the Lynden Bell statistics.

The next level of description i.e. the slow relaxation to thermodynamics equilibrium involving a small Fokker Planck term is treated by astronomers and plasma physicists with different philosophies. The plasma physicists consider mostly homogeneous plasma relaxing toward maxwellian distribution. The astronomers are specially interested in the evolution of the globular clusters; starting from a steady state solution of the Vlasov equation they let it change slowly through collisions.

Let us point out that this last problem raises very difficult questions, one of them being the possibility of time scale separation. Besides the question of the exact expression for a Fokker Planck term in an inhomogeneous medium (solved only for 
homogeneous neutral plasma through the Lenard Balescu operator) the solution of the problem implies an equation of the form

$$
\frac{\partial f}{\partial t}+\mathbb{V} f=\varepsilon \mathbb{F} f
$$

where $\mathbb{V}$ and $\mathbb{F}$ are respectively the Vlasov and Fokker Planck operator $(\varepsilon$ in front of $\mathbb{F}$ indicates that this operator is in principle very small). In the neutral homogeneous plasma the term $\mathbb{V}(f)$ is initially zero and remains zero although $f$ is slowly changing through the Fokker Planck term. This is of course not true for the globular cluster and multiple time scale technique should be used to solve (9). See Cole (1968).

Of course the direct integration of the $N$-body dynamical equation takes care of all these problems in principle. Nevertheless the problem of 'graininess' is still there. In all calculations the graininess effects are very much enhanced with respect to reality since the physical systems contain much more particles than the number which can be treated on the computer. If we are not interested in these 'graininess effects' we can try to decrease them. An interesting solution was introduced in plasma physics by Dawson and Hsi (1968) with the concept of 'cloud particles' i.e. of rigid structures of finite dimension larger than the interparticle distance but smaller than the wavelength of interest. On the other hand we can be interested in these graininess effects and we must know how they scale with $N$. This is a difficult question especially if we are interested in apparition of complex structures as the binaries stars where obviously triple correlation must play a role to allow the transition from open trajectories to close trajectories. These questions can be solved by careful comparison between computer simulation with different $N$ and a better understanding of the hierarchy of time scale for different effects - a very difficult theoretical problem.

Plasma physicists are actually very interested in 'graininess' effects since some astrophysical plasma and some of the laser created fusion plasma have a very high density. For some of these plasma $n D^{3}$ the number of electrons in the Debye cube can be of the order of unity or smaller. Although this problem has no equivalent in the self gravitating $N$-body problem it is worth mentioning the analytical and computer work on this question of one dimensional ('plane stars' with an uniform mass density) systems. The great advantage is the existence of analytical theory for canonical ensemble both for plasma (Lenard, 1961) and self gravitating system (Ribicky, 1972) and the possibility of performing exact computation (Feix, 1969) in the $2 N$ dimensional phase space for reasonably large time. It is the opinion of the author that such computations already initiated by Feix and Hohl (1968) will be extremely useful for a better understanding of foundations of statistical physics and its application to gravitational system (presence of isolating integral of motion, time average vs. ensemble average, difference for small $N$ between microcanonical and canonical ensemble, etc. ...).

\subsection{Collisionless EUlerian MODELS}

After this lengthy discussion of 'graininess' effects (but this is a central issue) we must mention that plasma physicists have developped models where they work directly 
with the Vlasov equation (representing a phase space fluid) although of course the Lagrangian superparticle model is still used to solve that equation with attempt to decrease the finite graininess effect through 'cloud particle' or filtering of high wavenumber structure of the electric field.

Eulerian model are interesting if we want to study the stability properties of steady state equilibria solution of the Vlasov equation. Up to now most of the results deals with one dimensional plane geometry, a model obviously more realistic and more popular in plasma physics than in stellar dynamics. Among some of the advantages of the Eulerian method is the fact that it is easier to make comparison with analytical theory (by turning on or off different terms which are neglected in the theoretical treatment). A discussion of the Eulerian model can be found in Chapters 2 and 3 of Alder and Fernbach (1970). Among Eulerian models a special mention should be made of the Multiple Water Bag. In Alder and Fernbach (1970), p. 88 Water Bag model is described. In this paper the philosophy is that we study a phase space fluid described by two levels of the possible phase space density. These two levels phase space density cannot of course describe the complexity of all possible velocity distribution but are sufficient te describe some of the strong nonlinear effects of two counterstreaming plasma. While Alder and Fernbach (1970) deals mainly with strong turbulence effects Doremus and Feix (1972) and Finzi et al. (1974) respectively for a self gravitating gas and a plasma study the possible steady states and their stability properties for one dimensional plane geometry.

Another point of view is introduced when we let the number of bags become large. Then we deal with a discrete element scheme for the Vlasov equation. Navet and Bertrand (1971) have shown that for finite time we recover all the properties of a continuous velocity distribution provided we take a sufficient number of bags. Moreover the time of validity increases linearly with this number. Very useful numerical properties are associated with this model, especially in the computation of properties of antennas immersed in an homogeneous plasma (a problem with no counterpart in stellar dynamics).

Indeed the Multiple Water Bag model is an extremely organised one where all the poles can be easily located and followed as a function of the different parameters. Details for grids and antennas problems can be found in Noyer et al. (1974).

\section{Results on Stability of Inhomogeneous Plasma Self Gravitating Gases and Vortex Equilibria}

\subsection{STEADY STATES SOLUTION FOR PHASE SPACE FLUIDS}

We sketch briefly results given in more details in Doremus and Feix (1972) and Finzi et al. (1974) and present the new analogy with the vortex problem.

Considering plane one dimensional geometry we know that the phase space density for a steady state is a function of the total energy $E$

$$
f=f(E)=F\left(\frac{1}{2} m V^{2}+\Phi\right)=F(E) .
$$


We will restrict our investigations to steady state of the double water bag type. Figure 1 precises the notation, it gives the velocity profile at point $x=0$ (we will suppose a symmetry around that point). We consequently have $V_{1}^{+}=-V_{1}^{-}=V_{1}$

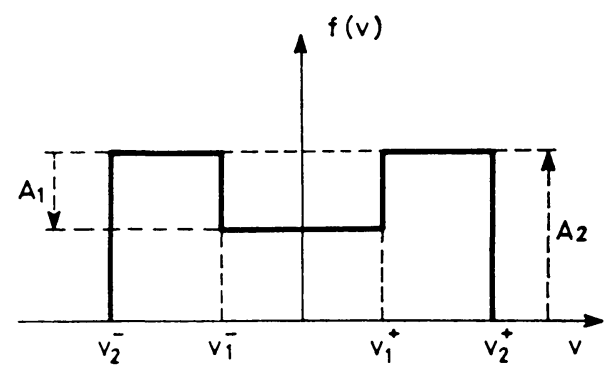

Fig. 1. The double 'Water Bag' distribution function with negative phase density of the inner bag.

$V_{2}^{+}=-V_{2}^{-}=V_{2}$ and define

$$
\beta=V_{1}^{2} / V_{2}^{2}, \quad \alpha=-A_{1} / A_{2} .
$$

Moreover at $x=0$ the potential energy is supposed to be minimum. For a self gravitating gas $\alpha$ and $\beta$ are the two parameters characterising the problem. We do not consider the case $A_{1}>0$ which is just a peculiar case of a decreasing $F(E)$ a case known to be always stable (Kulsrud and Mark, 1970; Feix et al., 1971). Consequently the parameter space is the square $0<\alpha, \beta<1$.
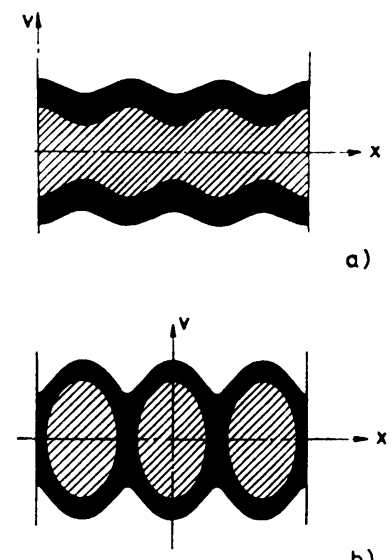

b)

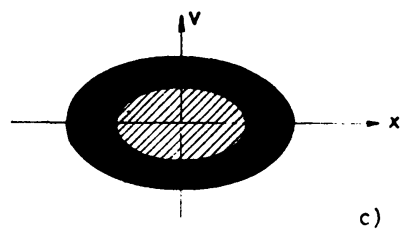

Fig. 2. Plasma. Phase space representation of the three possible types of steady states: (a) 'type 1' solutions (b) 'type 2' solutions, (c) 'type 3' solutions. 
Besides $\alpha$ and $\beta$ a third parameter $\gamma$ is needed to characterise the plasma, $\gamma$ is the ratio of ion density to electron density at point $x=0$. It can be shown that $\gamma$ must be bigger than 1 . In this problem ions are considered as motionless and providing a fixed, homogeneous background. This is a less academic case than it looks at first sight since it corresponds to an accelerator beam with one species of repulsing particles with a focusing force linear in $x$.

Figure 2 gives the three possibles type of steady states. Type 1 and 2 solutions are possible for a plasma only; type 3 for a plasma and a stellar gas. The existence of periodic nonlinear steady states is a characteristic of the plasma.
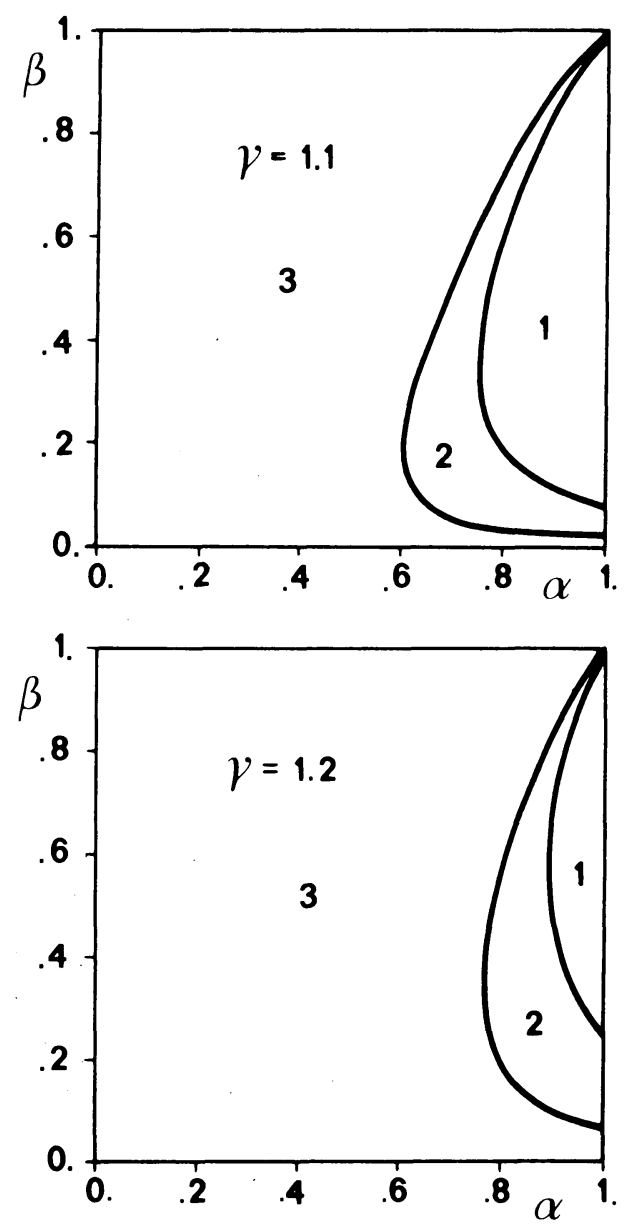

Fig. 3. Plasma.The $(\alpha, \beta)$ parameter space for $\gamma=1.1$ and $\gamma=1.2$. Locations of type 1,2 and 3 solutions.

Consequently any point of the square $0<\alpha, \beta<1$ is a possible steady state of type 3 for a self gravitating system. For a plasma we must consider the $\alpha, \beta$ square for a given $\gamma$. Figure 3 gives for $\gamma=1.1$ and $\gamma=1.2$ the limits of the three types solutions; when $\gamma$ 
increases it can be shown that region 1 and 2 tend to disappear simultaneously through the corner $\alpha=\beta=1$ and they do not exist any more for $\gamma>2$.

\subsection{STABILITY OF THE DIFFERENT STEADY STATES FOR PLASMA AND SELF GRAVITATING SYSTEM}

\subsubsection{Marginal Adiabatic Mode Theory}

Let us consider a steady state characterised by values of the parameters $\alpha, \beta, \gamma$. The question is to know if it is stable. If we consider type 1 solution we immediately recognize that we are in a case where two streams instability are likely to take place. From our knowledge of the homogeneous plasma case we guess that the instability will disappear through the point $\omega=0$. ( $\omega$ being imaginary and changing sign if we vary one of the parameter around its critical value). Since, close to the critical value, both the real and imaginary part of $\omega$ are small (for two streams the real part of $\omega$ is strictly zero) we call such a mode a marginal adiabatic (i.e. slowly varying) mode. Of course other modes can cross the stability - instability border in the $\omega$ plane around a finite value of $\omega$. Such modes called overstables must be also studied.

The theory of the marginal adiabatic mode has been given by Bertrand et al. (1972) and a detailed discussion is given in Doremus (1973) and Finzi (1973). If $A_{j}$ and $V_{j}(x)$ are the characteristic of bag $j$ the stability of the marginal adiabatic mode is given by the signs of the eigenvalues of the operator

$$
\left\{k^{2}(x)-\frac{\mathrm{d}^{2}}{\mathrm{~d} x^{2}}\right\} \Psi=\lambda \Psi .
$$

For a plasma with

$$
k^{2}(x)=\left(e^{2} / m \varepsilon_{0}\right) \sum_{j=1}^{N} 2 A_{j} / V_{j}(x)
$$

if all $\lambda$ are positive no marginal adiabatic mode is possible. If one is negative the system is unstable.

For types 1 and 2 solutions the boundaries conditions are $(\mathrm{d} \Psi / \mathrm{d} x)_{x= \pm \infty}=0$.

For type 3 solution the boundaries conditions are

$$
(\mathrm{d} \Psi / \mathrm{d} x)_{x= \pm L}=0
$$

where $\pm L$ are the space boundaries of the most external (most energetic) contour.

The interesting point is that (11) was recognized as already playing an important role in the solution of the steady state. Sticking to type 3 solution we can show that the steady electric field $E_{0}$ satisfies

$$
\left\{k^{2}(x)-\frac{\mathrm{d}^{2}}{\mathrm{~d} x^{2}}\right\} E_{0}(x)=0
$$


with $k^{2}(x)$ given by (12) as in (11). Boundaries conditions are now $\left(N_{i}\right.$ ion density)

$$
\mathrm{d} E_{0} / \mathrm{d} x=-e N_{i} / \varepsilon_{0} .
$$

The difference of boundaries conditions between (12) and (15) implies that none of the eigenvalues of (11) can be negative. To demonstrate this property we apply the Rayleigh Ritz method.

In such a method we compute

$$
R(\varphi)=\frac{\int \varphi A \varphi \mathrm{d} x}{\int \varphi^{2} \mathrm{~d} x}
$$

where $\varphi$ is a trial function satisfying the same boundaries conditions as $\Psi$ and $A$ is the operator $k^{2}(x)-\mathrm{d}^{2} / \mathrm{d} x^{2}$. If we can find that for such trial function $\int \varphi A \varphi \mathrm{d} x$ is always positive no marginal instability mode will appear. We introduce the trial function

$$
\varphi=\alpha(x) E_{0}(x)
$$

In (17) $\alpha(x)$ must be always defined. Indeed $E_{0}(x)$ for type 3 solution never cancels except for $x=0$ where we are free to take $\varphi=0$ (since $\Psi$ is a potential). Consequently $\alpha(x)$ has no discontinuity and we can integrate by part. Introducing (17) in $\int \varphi A \varphi \mathrm{d} x$ and taking (14) into account we find

$$
\left.\int \varphi A \varphi \mathrm{d} x=\int_{-L}^{L}\left(\frac{\mathrm{d} \alpha}{\mathrm{d} x}\right)^{2} E_{0}^{2} \mathrm{~d} x-\alpha \frac{\mathrm{d} \alpha}{\mathrm{d} x} E_{0}^{2}\right]_{-L}^{L}
$$

but for $x= \pm L$ we must have $\mathrm{d} \varphi / \mathrm{d} x=0$. Differentiating (17) we get

$$
\frac{\mathrm{d} \alpha}{\mathrm{d} x} E_{0}+\alpha \frac{\mathrm{d} E_{0}}{\mathrm{~d} x}=0
$$

and

$$
\left.\int \varphi A \varphi \mathrm{d} x=\int_{-L}^{L}\left(\frac{\mathrm{d} \alpha}{\mathrm{d} x}\right)^{2} E_{0}^{2} \mathrm{~d} x+\alpha^{2} E_{0} \frac{\mathrm{d} E_{0}}{\mathrm{~d} x}\right]_{-L}^{L}
$$

From the configuration at points $x= \pm L$ (where we find only ions since all the electrons are trapped inside this region) we see that $E_{0} \mathrm{~d} E_{0}(\mathrm{~d} x]_{x=L}$ is positive and $\left.E_{0} \mathrm{~d} E_{0} / \mathrm{d} x\right]_{x=-L}$ is negative; $(20)$ is positive and no marginal adiabatic mode can be present in type 3 plasma equilibria.

For type 3 self gravitating system it has been shown by Feix et al. (1971) that a marginal adiabatic mode always exists but that this mode is a trivial displacement mode and consequently does not correspond to a possible instability. The complete trapping of electrons or stars in type 3 equilibria precludes the existence of marginal adiabatic instability. 


\subsubsection{Overstable Modes}

Overstability for type 3 equilibria can of course occur. Skipping all details given in Doremus and Feix (1972), Finzi et al. (1974), Doremus (1973), and Finzi (1973) we just show in the parameter square $\alpha, \beta$ the zone of stability for type 3 equilibria. It is quite interesting to notice on Figure 4 (self gravitating system) and Figure 5 (plasma

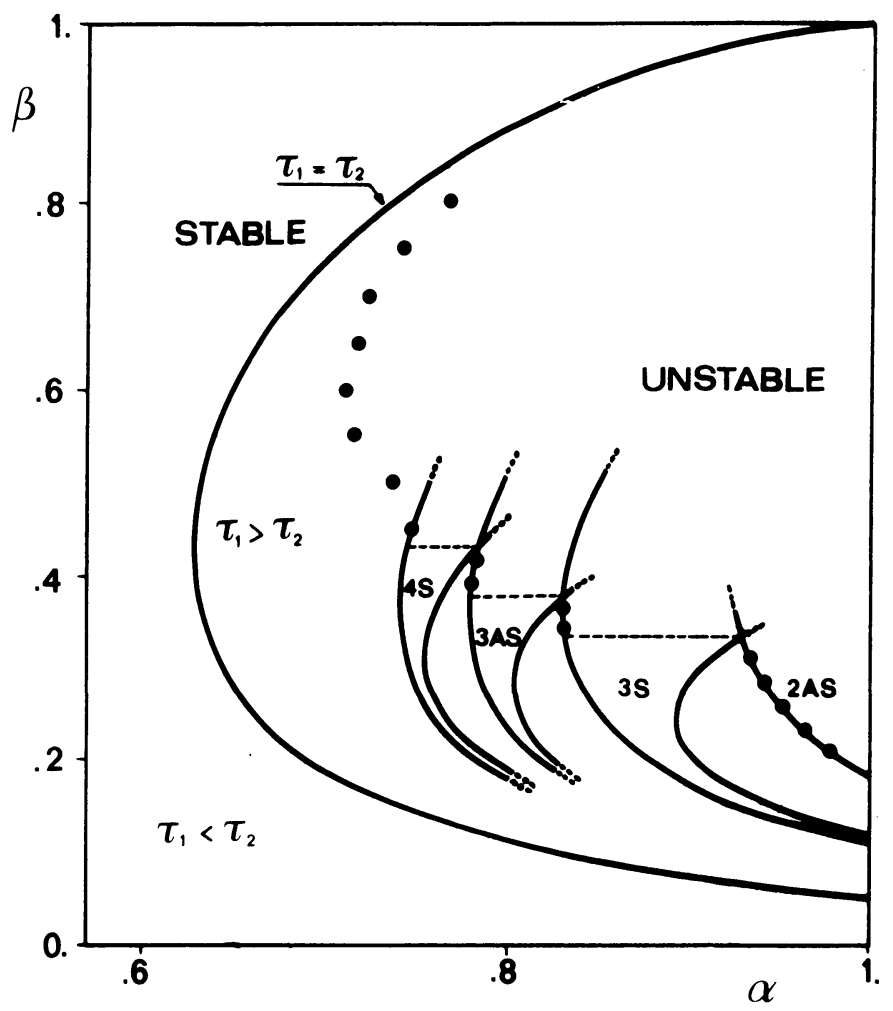

Fig. 4. Self gravitating systems : regions of instability in the $(\alpha, \beta)$ parameter space.

or accelerator beam) the similarity of the curves delineating the different unstable modes. Especially for the double water bag the region situated on the left of the curve $\tau_{1}=\tau_{2}$ (where $\tau_{1}$ and $\tau_{2}$ are the periods of oscillation of particles respectively on the inner and the outer bag) are stable although such a region corresponds to $\tau_{1}<\tau_{2}$ for self gravitating system and $\tau_{1}>\tau_{2}$ for plasma. In the lower part of the pictures the appearance of narrow bands of instability is a consequence of the two bags approximation and would 'disappear' in a completely continuous treatment. How such zones will evolve with a high bags number is an open question but preliminary results indicate that the filaments will become thinner and thinner and will in the limit constitute a nul measure set. 


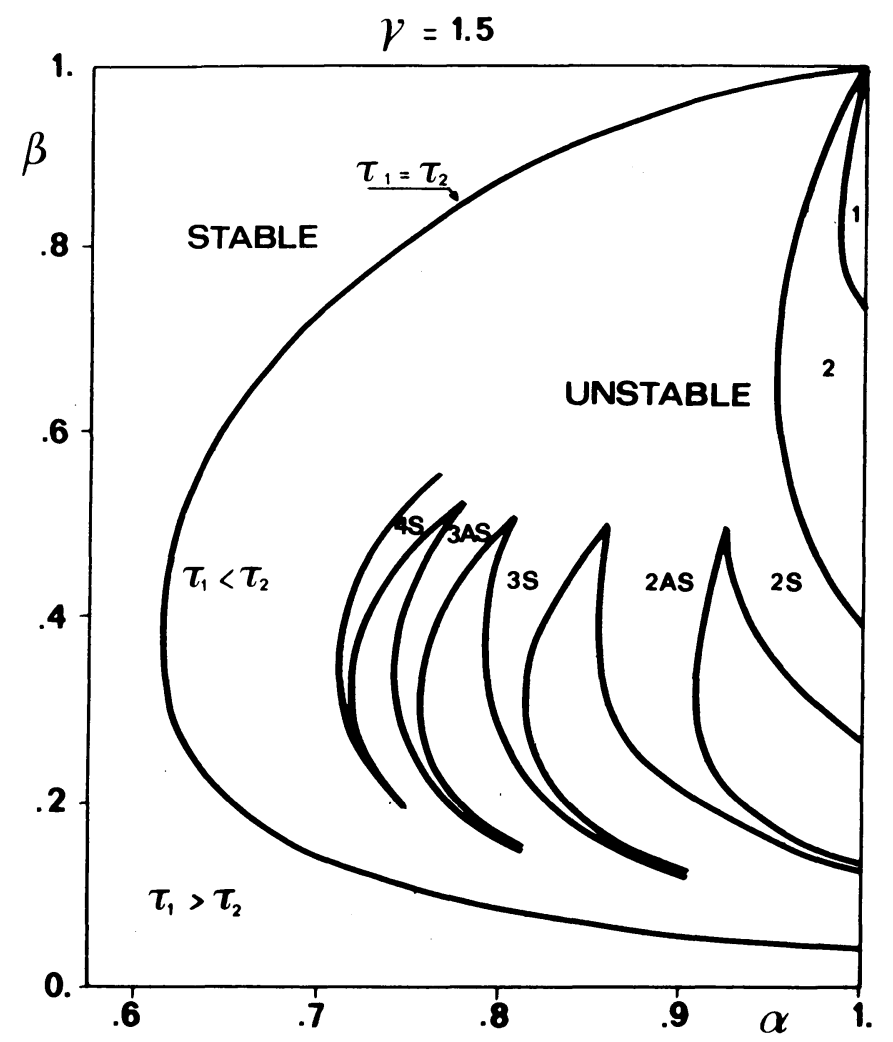

Fig. 5 Plasma. Regions of instability in the $(\alpha \beta)$ parameter space. $\gamma=1.5$.

\subsubsection{Type 1 and 2 Stability}

There is an interesting question since computer experiments (Finzi, 1972; Morse and Nielson, 1969) have shown that an initially homogeneous two stream unstable plasma evolves toward type 2 equilibrium and during sometimes such equilibrium were believed stable. But further computer run have exhibited a slow destruction of such equilibria through a coalescence of neighbouring holes. In this respect computer run involving both longer plasma and longer time are needed. For the two bags distribution we have shown (Feix, 1973) that all type 1 and most of type 2 equilibria are unstable. It is more than likely that all type 2 equilibria are in fact unstable but the considered trial functions of the Rayleigh Ritz methods are probably not general enough to treat all the cases.

\subsection{STABILITY OF VORTEX EQUILIBRIA}

We consider basically two kinds of equilibria corresponding to the following flows.

First a plane counterstreaming flow with a velocity $U_{y}(x, y)=0$ and $U_{x}(x, y)=U(y)$, $U(y)$ being an odd function in $y$. This corresponds to a vorticity profile which can be 
approximated by $N$ 'bags'

$$
\xi(y)=\sum_{j=1}^{N} A_{j}\left[H\left(y+y_{j}\right)-H\left(y-y_{j}\right)\right] .
$$

In (21) $H$ is the Heaviside function $H(x)=1$ if $x>0$ and $H(x)=0$ if $x<0$. A linearised analysis is carried on the motion of contours $y_{j}^{+}=y_{j}+\delta_{j}^{+}(x, t)$ and $y_{j}^{-}=-y_{j}+\delta_{j}^{-}(x, t)$; supposing a time space variation of $\delta_{j}^{+}$and $\delta_{j}^{-}$in $\exp i(\omega t-k x)$. We finally end up with an eigenvalues equation of the following type (we give a 3 bags example the generalisation of which is pretty obvious). We introduce the velocity values.

$$
\begin{aligned}
& U_{1}=\left(A_{1}+A_{2}+A_{3}\right) y_{1} \\
& U_{2}=A_{1} y_{1}+\left(A_{2}+A_{3}\right) y_{2} \\
& U_{3}=A_{1} y_{1}+A_{2} y_{2}+A_{3} y_{3}
\end{aligned}
$$

and suppose that the velocity profile is increasing monotonically from $-U_{3}$ to $U_{3}$, i.e. that all $A_{j}$ are positive.

$$
\begin{array}{llllll}
1-\frac{2}{A_{1}}\left(\omega+k u_{1}\right) & {[1+1]} & {[2-1]} & {[2+1]} & {[3-1]} & {[3+1]} \\
{[1+1]} & 1+\frac{2}{A_{1}}\left(\omega-k u_{1}\right) & {[2+1]} & {[2-1]} & {[3+1]} & {[3-1]} \\
{[2-1]} & {[2+1]} & 1-\frac{2}{A_{2}}\left(\omega+k u_{2}\right) & {[2+2]} & {[3-2]} & {[3+2]} \\
{[2+1]} & {[2-1]} & {[2+2]} & 1+\frac{2}{A_{2}}\left(\omega-k u_{2}\right) & {[3+2]} & {[3-2]} \\
{[3-1]} & {[3+1]} & {[3-2]} & {[3+2]} & 1-\frac{2}{A_{3}}\left(\omega+k u_{3}\right) & {[3+3]} \\
{[3+1]} & {[3-1]} & {[3+2]} & {[3-2]} & {[3+3]} & 1+\frac{2}{A_{3}}\left(\omega-k u_{3}\right)
\end{array}
$$

where $[i \pm j]$ are short notations to designate the functions $\exp \left(-k\left|y_{i} \pm y_{j}\right|\right)$.

It can be shown that the poles go by pair $\omega$ and $-\omega$ and that their motions is described on Figure 6.

For $k \rightarrow \infty$ phase velocities are $\pm U_{1}, \pm U_{2}$ and $\pm U_{3}$; then all roots remain located between two neighbouring velocities except for the first one which goes through the value 0 for a given $k=k_{c}$ and becomes imaginary for $k<k_{c}$ introducing an instability through a marginal 'adiabatic mode' in close analogy with the type 1 and type 2 equilibrium.

It is now interesting to see what happens when we take instead of a plane flow a rotating one corresponding to a cylindrically symmetric equilibrium with a vorticity 
distribution $\xi$ ( $r$ is the radial coordinate)

$$
\xi=\sum_{j=1}^{N} A_{j} H\left(r_{j}-r\right) .
$$

The case $N=1$ corresponding to the Rankine vortex.

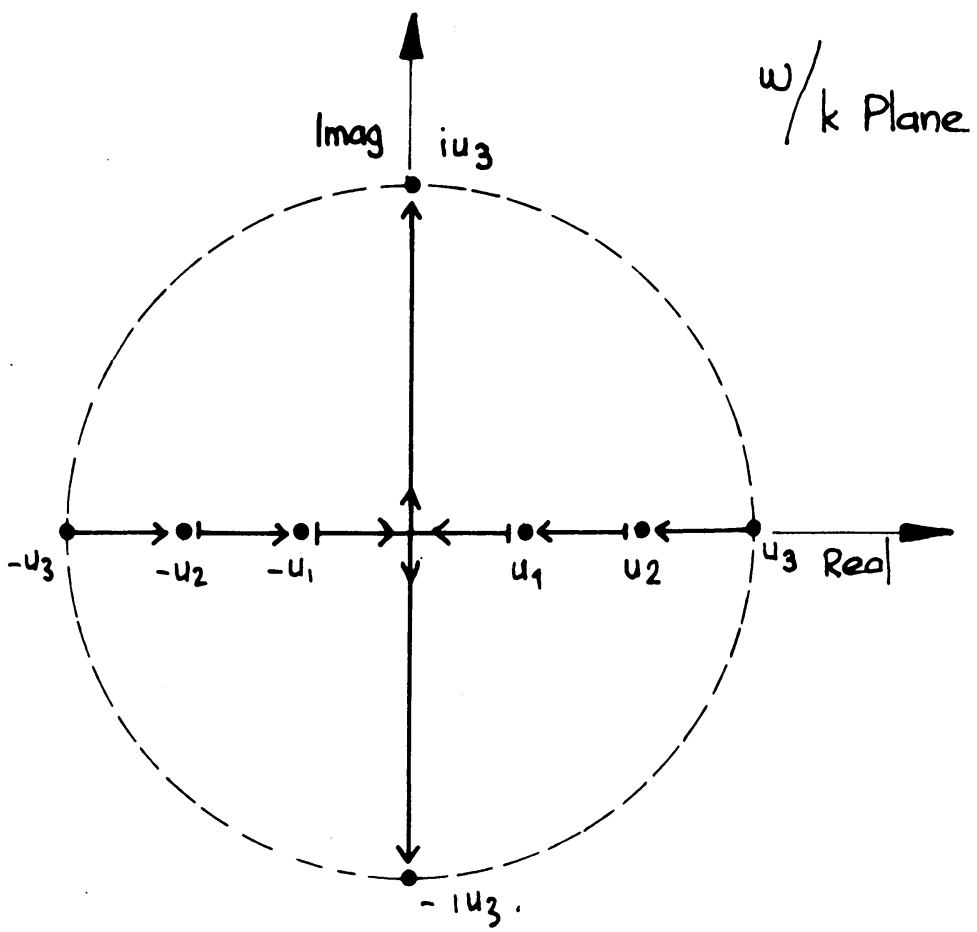

Fig. 6 Vortex Fluid. Plane geometry. Evolution of the phase velocities in the $\omega / k$ plane. Illustration for 3 bags with all $A_{j}>0$.

Again we suppose all the $A_{j}>0$ and notice the corresponding velocity profile

$$
U_{i}(r)=\sum_{j=1}^{i-1} \frac{A_{j}}{2} \frac{r_{j}^{2}}{r}+\sum_{j=i}^{N} \frac{A_{j}}{2} r
$$

for $r_{i-1}<r<r_{i}$.

A linearized motion around the equilibrium is studied with

$$
r_{i}(\theta, t)=r_{i}+\delta r_{i} \exp i(\omega t-m \theta)
$$

$m$ taking an integer value. The eigenfrequencies matrix becomes

$$
\begin{array}{lll}
1+\frac{2}{A_{1}}\left(\omega-m \Omega_{1}\right) & {[1,2]_{m}} & {[1,3]_{m}} \\
{[1,2]_{m}} & 1+\frac{2}{A_{2}}\left(\omega-m \Omega_{2}\right) & {[2,3]_{m}} \\
{[1,3]_{m}} & {[2,3]_{m}} & \left.1+\frac{2}{A_{3}} \omega-m \Omega_{3}\right),
\end{array}
$$


where $[i, j]_{m}$ stands for $\left(R_{i} / R_{j}\right)^{|m|}$ if $R_{i}<R_{j}$ or $\left(R_{j} / R_{i}\right)^{|m|}$ if $R_{i}>R_{j} \cdot \Omega_{1}, \Omega_{2}, \ldots, \Omega_{N}$ are the angular frequency of the fluid element on contour 1,2 , etc.... We have

$$
\begin{aligned}
& 2 \Omega_{1}=A_{1}+A_{2}+A_{3} \\
& 2 \Omega_{2}=A_{1}\left(\frac{R_{1}}{R_{2}}\right)^{2}+A_{2}+A_{3} \\
& 2 \Omega_{3}=A_{1}\left(\frac{R_{1}}{R_{3}}\right)^{2}+A_{2}\left(\frac{R_{2}}{R_{3}}\right)^{2}+A_{3} .
\end{aligned}
$$

The positiveness of the $A_{i}$ implies that $\Omega_{1}>\Omega_{2}>\Omega_{3}>\cdots>\Omega_{N}$. Now in analogy with the plane flow case we study the behaviour of the eigenfrequencies when $m$ goes from $+\infty$ to 1 . Now all the poles can be shown to be on the real axis and if we look at the $\omega / m$ axis the angular phase velocities can be shown to vary from $\Omega_{1}, \Omega_{2}$ and $\Omega_{3}$ for $m=+\infty$ to $\Omega_{2}, \Omega_{3}$ and 0 for $m=1$. Figure 7 . The marginal $m=1 \omega=0$ mode is simply

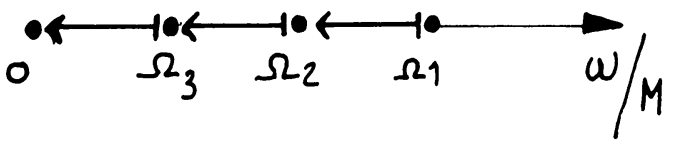

Fig. 7 Vortex Fluid. Cylindrical geometry (Generalised Rankine Vortex). Evolution of the angular phase velocity on the $\omega / m$ axis. Illustration for 3 bags with all $\boldsymbol{A}_{j}>0$.

a displacement mode for the total vortex and consequently no instability will occur through the point $\omega=0$. If all the $A_{j}$ are positive the flow will be stable but if the vorticity profile exhibit a minimum, overstability can occur. In analogy with the plasma case the complete 'trapping' of the vorticity distribution has killed the marginal unstable adiabatic mode which just becomes in the limit $m=1$ a trivial displacement mode. We notice here that the bidimensional vortex distribution present analogies both with the plasma and the stellar system. The infinite plane system with no stellar dynamic counterpart exhibiting unstable roots while the cylindrical one as the self gravitating gas exhibit a displacement mode (while the plasma case has a $\omega=\omega_{p}=$ $=\left(n_{i} e^{2} / m \varepsilon_{0}\right)^{1 / 2}$ mode corresponding to a global oscillation of the electron population in the neutralising fixed ion background.

\section{Conclusion}

Plasma physicists and astronomers face the same $N$-body long range force problem. But while the plasma machines impose certain symmetry which can simplify the computations the astronomers attack the complete $N$-body problem taking into account both short range and long range interaction. As a consequence the plasma physicists should look more carefully into these binaries phenomena that he has a certain tendency to neglect (hydrodynamics is full of problems where the existence of a small viscosity coefficient brings very singular phenomena often presented as paradox). May be the Vlasov equation for some geometries (spherical particularly) will 
also exhibit singular behaviour. Now that long confinement time machines are under construction methods to compute the long time behaviour of our plasma will be needed (including collision, recombination and ... hopefully fusion). The astronomer's Monte-Carlo method is obviously a candidate.

The plasma physicists on the other hand have developped Eulerian schemes very efficient for the stability studies (as the Multiple Water Bag and the various models involving Fourier, Hermite and Tchebycheff transforms on configuration and velocity spaces). The advantage of such models is that the analysis can be carried further on, simplifying the numerical work (the Multiple Water Bag model being a good example).

May we invite the astronomers also to get out of the real world to play with one dimensional and two dimensional models (not to mention the $1 \frac{1}{2}$ )? Especially if one wishes to check a tricky hypothesis, sometimes the analytical computation and the numerical simulation can be very much simplified without modifying the essence of the approximation. A good example is the computation of correlation properties in plasma where concept as the test particle is completely independent of the dimensionality (but results do depend of it).

To end this paper I. will make some propaganda for a general study of models and especially of fully discretised models. The Multiple Water Bag is a first step but is not discretised in space neither, of course, in time.

We should study what are the qualitative properties (for example stability properties) which are retained in the discretisation process.

Many questions are still opened for mathematical physicists: numerical analytical method, multiple time scale system of differential equations, use of Formal algebraic manipulating language, etc.....

After many years where new interesting results were rather easily obtained at the beginning of computational physics it may be time to get new and more powerful tools!

\section{Acknowledgements}

The author thanks for fruitful discussions on these topics Drs E. Jamin, M. Navet and M. L. Noyer. The work on the vortex stability has been done in cooperation with them.

\section{References}

Alder, B. and Fernbach, S.: 1970, Methods in Computational Physics, Vol. 9, Academic Press, New York, London.

Bertrand, P., Dorémus, J. P., Baumann, G., and Feix, M. R.: 1972, Phys. Fluids 15, 1275.

Christiansen, J. P. and Zabusky, N. J.: 1973, J. Fluid Mech. 61, 219.

Cole, J. D.: 1968, Perturbation Methods in Applied Mathematics, Blaisdell, New-York.

Dawson, J. M. and Hsi, C. G.: 1969, Proceeding APS Topical Conference on Numerical Simulation of Plasma, LA 3990, Los Alamos.

Dorémus, J. P.: 1973, 'Contribution à l'étude de la stabilité des fluides de l'espace des phases', Université de Nancy I (Thèse de Doctorat).

Dorémus, J. P. and Feix, M. R.: 1972, Astron. Astrophys. 20, 259.

Feix, M. R.: 1969, in G. Kalman and M. R. Feix (ed.), Non Linear Effects in Plasmas, Gordon and Breach. New York, London, Paris, p. 151. 
Feix, M. R.: 1971, in M. Tilston and M. Sauzade (eds.), Progress in Radio Science, URSI, Bruxelles, Vol. 3, p. 271.

Feix, M. R.: 1973, Some Problems and Methods in Computational Plasma, Culham SRC Symposium on Turbulence and Non Linear Effects in Plasma, to be published.

Feix, M. R., Dorémus, J. P., and Baumann, G.: 1971, Astrophys. Space Sci. 13, 478.

Feix, M. R. and Hohl, F.: 1968, Bull. Astron., Ser. 3, 2, 289.

Finzi, U.: 1972, Plasma Phys. 14, 327.

Finzi, U.: 1973, 'Instabilité des Structures non linéaires dans les plasmas: ondes BGK', Université de Paris XI (Thèse de Doctorat).

Finzi, U., Dorémus, J. P., Holec, J., and Feix, M. R.: 1974, Plasma Phys. 16, 189.

Kulsrud, R. M. and Mark, J. W. K.: 1970, Astrophys. J. 160, 471.

Lénard, A.: 1961, J. Math. Phys. 2, 682.

Morse, P. M. and Feshbach, H.: 1953, Methods of Theoretical Physics, McGraw Hill, New-York.

Morse, R. L. and Nielson, C. W.: 1969, Phys. of Fluids 12, 2418.

Navet, M. and Bertrand, P.: 1971, Phys. Letters 34A, 117.

Noyer, M. L., Navet, M., and Feix, M. R.: 1975. 'The Multiple Water-Bag Model for Forced Oscillations in a Warm Isotropic Plasma', J. Plasma Phys. (in press).

Ribicky, G. B.: 1972, Astrophys. Space Sci. 14, 56.

Zabusky, N. J. and Deem, J. S. : 1971, J. Fluid Mech. 47, 353.

\section{DISCUSSION}

Ipser: In the unstable case you talked about, involving the inverted distribution as a function of energy, are you dealing with spherical systems?

Feix: No. It is a plane problem and this is an overstable instability (which means that the instability appears through an $\omega \neq 0$ mode).

Severne: One could mention another domain of plasma physics which may prove very directly relevant to stellar dynamics. In the study of laser induced fusion, one has to analyse spherical systems in strong fields and which are strongly inhomogeneous.

A comprehensive theoretical program has been initiated at the Université Libre de Bruxelles.

Feix: O.K. Let us hope we will get money for such studies ! 